\title{
Energy Efficient Routing Protocol Avoiding Route Breaks based on DSR
}

\author{
Ashish Kumar \\ Dept. of CS\&E, CET \\ IFTM University, Moradabad
}

\author{
M. Q. Rafiq \\ Professor, Dept. of CE \\ AMU, Aligarh
}

\author{
Kamal Bansal \\ UPES \\ Dehradun
}

\begin{abstract}
An accelerating energy crisis in the oil and gas industry is driving development and investment in Mobile Ad Hoc Network technologies. The mobile ad hoc network (MANET) is a self-configuring infrastructure-free network of mobile devices connected by wireless links; it is essentially a temporary wireless network which users who are willing to communicate form and who subsequently use multi-hop peerto-peer routing to provide the network connectivity. The nodes in MANET being mobile may result in dynamic topology with a high rate of link breakages and network partitions leading to an interruption in the ongoing communication. The link breaks in MANET routing protocols are significant contributor of energy consumption. In order to reduce the cost incurred in tackling the link failure, a routing protocol avoiding route breaks based on Dynamic Source Routing (DSR) has been proposed. The proposed energy aware efficient DSR (EAEDSR) considers the link and node stability to determine preemptive metric. The protocol's performance is found to be promising.
\end{abstract}

\section{General Terms}

Energy Consumption in MANET, Oil and Gas Industry.

\section{Keywords}

MANET, Link breaks, Routing Protocols, DSR, AODV and DSDV etc.

\section{INTRODUCTION}

A MANET [1,2] is a self organized network of mobile nodes connected by wireless links with no existing pre-established infrastructure for communication. The nodes can move freely and in arbitrary manner. All nodes within the range of each other can communicate without the need of a central access point. Each node can act as both a router and as a host for multihop messages. The nodes in the network forward messages on behalf of other nodes which are not in the transmission range of each other. Hence separate schemes are required for such dynamically changing network. It is anytime, anywhere type of network. Because of this such a network can be quickly deployed in emergence services such as disaster recovery like fire and search and rescue operation, and the ease of data acquisition in inhospitable terrains makes it suitable for military operations.

An accelerating energy crisis in the oil and gas industry is driving development and investment in MANET technologies. MANET is a key investment area across the whole oil and gas industry. The importance of MANET in oil and gas industry can be understood from the following: The availability of crude oil is in the remote area of seas and there it is very difficult to implement infrastructure oriented network for communication, sometimes the working staff faces catastrophic situations in such remote areas and it is very difficult for them to communicate with each other without any infrastructure, hence required a infrastructure less network (MANET). This is not the only sector; many other projects are going on for its success with huge investment.

MANET was designed to work in the situations such that whenever certain number of nodes come together i.e. within transmission range of each other, they should be able to communication. Its aim was not as for the fixed network i.e. not for long time. So generally in MANET nodes are equipped with low power and computing, having routing capabilities. Because, in MANET, topology frequently changes, and nodes have got limited power so its routing is challenged by these factors. So conventional routing protocols employed for wired networks cannot be used.

Initial routing protocol considered minimum number of hops as metric, but the problem of overuse of nodes on a path may result in disruption in communication. It is very serious problem consider the rescue operation where all nodes need to be connected and the overuse may lead to network partitions and the communication may no longer be maintained. Hence this poses serious concern.

\section{RELATED WORKS}

In recent years, several wireless routing protocols have been designed to provide uninterrupted communication. These protocols have to adapt quickly to the changes in the network. And more ever route disconnection has a serious negative effect on packet delivery and performance. A technique to improve delay caused due to link failure resulting in route rediscovery has been proposed in [3]. In the technique the power levels of the nodes are computed. And during a transmission whenever the power level of the node $\mathrm{N}$ (likely to cause link failure) goes below a certain threshold value, it sends warning message to predecessor of $\mathrm{N}$ for determining an alternative path so that remaining packets can be transmitted through alternative path. The DSR protocol performance under preemptive routing has been evaluated in [4]. To generate warning message required to start the discovery of an alternative path the signal strength threshold is used. And it is based on the concept of preemptive region width given in [5]. In [6] author proposed enhanced power based multipath routing protocol. In the technique if the signal strength falls below threshold value triggering warning message being sent to source node. And source node has multiple paths to destination being found during initial route discovery phase. The [7] proposed the preemptive multipath ad hoc on demand distance vector routing (PMAODV) based on the concept of AODV with multipath routing and 
preemptive routing protocol. [8] Improves AODV by adding the concept of preemptive routing and proposed the predictive preemptive AODV (PPAODV) routing technique. When a node moves to preemptive region, its signal strength falls below threshold. So to predict when to send warning message it uses three consecutive signal strengths of the packets received from predecessor in Lagranges interpolation formula i.e. it measure $\mathrm{P}_{0}, \mathrm{P}_{1}$ and $\mathrm{P}_{2}$ at $\mathrm{t}_{0}, \mathrm{t}_{1}$ and $\mathrm{t}_{2}$ respectively and put the values in Lagranges formula [8] to compute received signal strength (formula describes in paper in detail) P. When $P$ falls below threshold value, a warning message is sent to predecessor and it starts local route repair by finding alternative path to destination. Author evaluated protocols performance on Glomosim simulator. [9] Proposed Cacheenabled Preemptive Dynamic Source Routing (CPDSR) technique. This method suggests the solution to the problem of stale routes available in nodes caches. As preemptive routing produces early warning so RERR packets are not being broadcasted hence nodes are still with that stale route information in their cache. The warning node send warning message to source node in turn source node refreshes its local route cache and finds new available route in its cache to destination if exist then uses that route and if not then broadcast RREQ packets with unstable link (likely to break soon) information to find the new route. Unstable link information is kept in RREQ so that all the nodes on the path may refresh their cache with this and any new replied route does not contain such unstable link. After this source node sends RERR message to the warning node so that all the nodes on this path may refresh their route cache and warning node may know that route has changed. The author has also proposed a method to determine reaction time. It is the time to determine when a link is regarded as unstable.

The [10] presented a technique to hand-off the router in case of weak link. It is determined by considering the power difference table and neighbor power list which is kept by every node. Based on these tables values warning node determines neighbor node which is reachable from source and destination node both so that the data can be diverted through that path and warning node broadcast a hand-off packet containing the address of predecessor and successor to make the path. If the neighbor node with sufficient link stability cannot be determined then allow the link fail to occur and later route repair is done as per AODV protocols standard mechanism. [25] Gives an exhaustive survey of the various techniques dealing with the link failure and overhead of routing protocols.

\section{PROBLEM ISSUE}

The nodes in MANET are dependable upon the exhaustible power supply. Also because of shared nature of medium, the transmitted packets may also cause energy loss in the surroundings nodes due to overhearing. And compared to wired network there is increased possibility of packet loss and congestion in MANET resulting in energy consumption. Energy consumption can also be due to receiving of data, transmitting the data, traffic, mobility and size of the network. While the problem of network partitioning due to the movement of nodes cannot be handled by routing protocol, partitioning due to outage of battery can be solved by routing decisions. Routing techniques helps in path establishment for communication. MANET consists of different types of overheads such as routing overhead (route request, route reply and route error packets). The overhead caused in managing the link failure is a significant contributor of energy consumption. As the start node of the broken link has to wait/ retry for a time out interval before deciding that the link is failed and cannot be used further and has to inform through ROUTE ERROR (RERR) packet to all other nodes using the failed link in their path. Also the packets following this path experience large delays and the source node has to find a new route to destination. And this problem occurs more frequently in wireless networks. Hence there are two major reasons of link breakage in MANET:

a) Nodes energy getting depleted with time

b) Nodes moving out of the transmission range of neighbor

Hence in order to reduce the cost incurred in managing the link breaks we should determine the stable routes. The protocol in [17] considers energy expenditure in data transmission to route the data, but the lifetime of the node and link expiration time is not considered [12, 13, and 14]. Most of reported research work in this area attempt either considering node stability, route stability, energy spent in data transmission or link disjoint paths [26] however to the best of my knowledge none has reported a technique considering energy aware node stability, path quality in terms of link stability, traffic aware and data types (real time and non real time) all into a single routing protocol. We present a technique based on preemptive routing [5] to reduce link breaks and routing overhead for MANET based on DSR. The technique works for both data types i.e. real time and non real time data.

\section{PROPOSED APPROACH}

The link and node's stability is measured using two metrics, the link expiration time (LET) [15] and remaining energy of the node. These metrics are combined to generate a measure which will reduce the cost of handling the link breaks. Because in normal condition the routing protocol searches for alternative path only after the current path fails and the cost of failure detection is high since many retries have to time out before being pronounced dead [5]. The proposed measure will help to keep the cost low.

\subsection{Radio Propagation Model}

According to NS2 implementation Two Ray Ground Reflection Approximation is used as radio propagation model. The signal power strength at the receiver is:

$P_{r}=\frac{P_{t} \times G_{t} \times G_{r} \times\left(h_{t}^{2} \times h_{r}^{2}\right)}{d^{4}}$

Where:

$P_{r}$ is the signal power at the receiver

$P_{t}$ is the signal power at the transmitter

$G_{t}$ is the gain for signal to a node from the transmitter

$G_{r}$ gain for a signal to a node from the receiver

$H_{t}$ is height of transmiiter antenna

$H_{r}$ is height of receiver antenna

$d$ is the distance between transmitter and receiver 
Equation (1) can be simplified [18] under the conditions of ad hoc wireless network simulation to:

$$
P_{r}=k \frac{P_{t}}{d^{4}}
$$

Where:

$$
k=G_{t} \times G_{r} \times\left(h_{t}^{2} \times h_{r}^{2}\right) \text { is a constant. }
$$

\subsection{Predicting Link Expiration Time}

Link Expiration Time (LET): The link expiration time prediction algorithm based on signal strength of receiving packets was proposed in [22]. But the algorithm assumes that the nodes are moving in the same direction with same speed. But being ad hoc in nature the prediction based on speed and direction may not be accurate. We are proposing LET prediction measurement based on the signal strengths of the receiving packets. According to the Fig 1, node $B$ receives the signals from node $A$ at time $t_{o}, t_{1}$ and $t_{2}$. Suppose the strength of the received signals is $P_{0}, P_{1}$ and $P_{2}$ respectively. The signal power threshold, for the wireless interface is fixed. Suppose node $B$ receive the packet at time $t$ with signal strength equivalent to threshold $P_{s}$, then using the Lagrange interpolation the predicted LET $(\mathrm{t})$ is given by

$t=\frac{\left(P_{s}-P_{0}\right)\left(P_{s}-P_{1}\right)}{\left(P_{2}-P_{0}\right)\left(P_{2}-P_{1}\right)} t_{2}+\frac{\left(P_{s}-P_{1}\right)\left(P_{s}-P_{2}\right)}{\left(P_{0}-P_{1}\right)\left(P_{0}-P_{2}\right)} t_{0}+\frac{\left(P_{s}-P_{0}\right)\left(P_{s}-P_{2}\right)}{\left(P_{1}-P_{0}\right)\left(P_{1}-P_{2}\right)} t_{1}(3)$

We have used the power strengths of the three signals and their time of occurrences. If the power strength of the two consecutive measurements remains same then we consider the one with the later time. We have also assumed that nodes are able to measure the strength of the received signals.

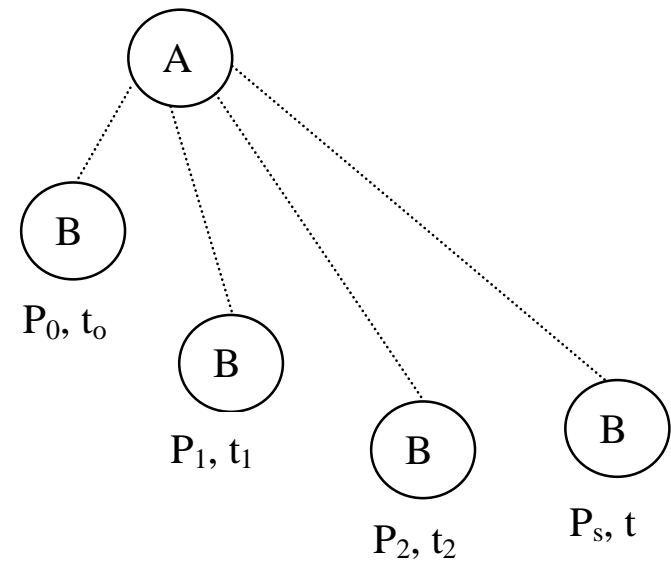

Figure 1: Movement of a node at different times

\subsection{Node Stability}

The power consumption at the network layer is because of two operations namely communication and computation [23]. The communication power is consumed due to transmission and reception of packets of information. The power is also discharged even the node is idle and waiting for the packets. The computation power refers to the power spent in calculations that take place inside the node for routing and power adjustments etc. Energy consumption of a node at time $t$ is taken as given by following equation (4).

$$
E_{c(t)}=N_{t} * p+N_{r} * q
$$

$E_{c(t)}$ is the energy consumed by a node after time $\mathrm{t}$

$N_{t}$ is the number of packets transmitted by a node

$N_{r}$ is the number of packets received by a node

$p$ and $q$ are constants factors, depends on the energy model [27] used

Thus if $E_{i}$ is the initial energy of a nodes, then remaining energy of a node after time $\mathrm{t}$ is given by equation (5)

$$
E_{r(t)}=E_{i}-E_{c(t)}
$$

The main objective of the work is to find the stable path from source to a given destination and thereafter reducing the efforts required in handling the link breaks. The path stability is based on the stability of links on the path. In order to find the stable path we have considered received signal power strength and traffic level as the main criterion. The stable path will reduce the number of link breaks resulting in cost reduction otherwise which have been incurred in handling the failures.

\subsection{Link Stability Measure (LSM)}

It can be inferred that link stability is directly proportional to Link Expiration Time because higher value of LET will increase the link duration (availability). And it is also proportional to nodes stability as higher energy balance of the node will improve network lifetime. Hence we have taken Link Stability Measure (LSM) as the product of both the LET and NS as given in equation (6).

$$
\text { Link Stability Measure }(L S M)=L E T \times N S
$$

The $L S M$ value determines the network performance. We have used LSM as the preemptive measure in the modified DSR. We have chosen the Dynamic Source routing (DSR) [17] protocol as a candidate protocol. The proposed Energy Aware Efficient DSR (EAEDSR) works in three phases.

\section{Route Discovery \\ 2. Route Selection \\ 3. Route Maintenance}

The path discovery is started by sending Route Request (RREQ) packets to all its neighbors satisfying broadcasting condition. The RREQ packets has been modified to carry the Node's stability (NS), traffic level (TL), weak nodes (WN) and type of data (real time traffic and non-real time traffic) in addition to standard information. The traffic level field carries 
the cumulative traffic of the nods on the paths followed by the RREQ packet and initially by default the TL value is zero. The Figure 2(a) shows the RREQ packet format. The initial value of $L S M$ is zero at every node and it is updated as the data transmission goes through the node over the path. Every node also maintains a table called Neighbor Information Table (NIT). Figure 2 (b) shows the structure of NIT.

The proposed scheme does not allow source node to maintain route cache for long time as the network conditions changes rapidly in terms of energy levels of nodes. We have modified the DSR to force it to periodically refresh its cache and trigger a new route discovery every 10 seconds to better reflect the present power condition of the nodes so that the same may be reflected in link stability measure. When the source node refresh its cache the last predicted LET values of nodes on the path is used till the new one is computed. The nodes may know about the energy balance of their neighbors from RREQ packets.

Src Dstn Type ID TTL Hops NS ToD WN TL Path

(a)

Src Dstn ID Hops RP TL

(b)

\section{Figure 2: RREQ packet format and NIT table format}

\section{MODIFIED DSR (EAEDSR)}

\subsection{Route Discovery and Selection}

In DSR when the source node needs to send the packets to a destination node, starts a route discovery by sending RREQ to all its neighbors. Upon receiving RREQ, a node checks whether its own address is listed in the path list of received RREQ, if its address is present, the packet is dropped. Otherwise the node appends its own address to the list of path and before rebroadcast to all its neighbors waits for a period, till the subsequent packets (RREQs) with the same identification number traveled through other paths have been received. As RREQ reaches a node, it enters its information i.e. traffic level and received power (RP) in NIT table. Because RREQs have been traveled through different paths hence may differ in link stability measured by received power signal strengths. For broadcasting only the neighbors having Traffic Level <=TL are selected. As the wait period expires, the requests with RP values > thresholds are selected for broadcasting and node's traffic level value is also added to the packet. Intermediate nodes on the path are not allowed to send RREP, from their local cache. This may result in a route, which do not fulfill considered requirements needed to maintain the performance. The destination receives the multiple RREQ, and it selects the paths with disjoint nodes. If the type of data received is real time traffic then it selects the path with the minimum number of weak nodes and unicast the reply. The source can start transmitting the data. Otherwise i.e. in case of non real time traffic the destination generates multiple replies and unicast them to the source. Now source has multiple node disjoint paths to the destination. The source sorts the path in the increasing order of the number of weak nodes. In case of multiple paths with the same number of weak nodes, sorts them in the increasing order of traffic levels. If the traffic level is also same then the path with the minimum number of hops is selected.

\subsection{Route Maintenance}

During the transmission if the LSM falls below LSM $_{\mathrm{thr}}$, it informs to its predecessor node about node failure by sending NODEFAIL message. Once a node receives such a message it sends ROUTEFAIL message to the source node. The source can then reroute the packets by backup routes, if available. If no backup's route exists, then source starts the route discovery procedure again. If the multiple routes found during discovery are used as back up routes on one by one basis during primary route failure then a significant net gain in probability of breakage may be obtained [24].

$$
\text { Net Gain }=P_{p r}-P
$$

Where $P_{p r}$ is the primary route breakage probability and $P$ is the multiplicative probability of backup routes. Since the routes are independent of each other being nodes disjoint. And it has been proved that the performance of the DSR with backup route approach is better than the conventional DSR. But the problem with the backup route approach is that while the source is using the one route the other route might fail and source may remain unaware of that and will pose a problem. To overcome this, the use of multiple routes simultaneously will improve the communication between two ends. And it has been prove that the use of multiple routes simultaneously i.e. in parallel will reduce the probability of communication breakage exponentially.

\subsection{Simulation and Results}

The protocol is simulated using ns 2 [20]. The simulation area is of 500 x 500 sq. m size, where 25 nodes are placed. The selection of source and destination is done on random basis. The channel model used is free space. The traffic sources used in the simulations generated constant bit rate (CBR) data traffic. The energy model taken is as used by [21]. The values used correspond to 2,400 MHz Wave LAN implementation of IEEE 802.11. The radio frequency value is set as $0.2818 \mathrm{~W}$ for transmission range of $250 \mathrm{~m}$. A packet size of 512 bytes is used. The nodes move according to the random way point mobility model. The nodes move with a speed of $5.0 \mathrm{~m} / \mathrm{s}$. The packet sending rate is 4 packets/ sec. The initial energy of the nodes is 1000 Joule. The power consumed in transmitting and receiving the packets is $1.65 \mathrm{~W}$ and $1.15 \mathrm{~W}$ respectively. We have taken power consumption due to idle and sleep condition into consideration and it is $1.0 \mathrm{~W}$ for idle condition and 0.001 $\mathrm{W}$ for sleep state. We have assumed that link breaks occur 
when receiving power is below the receive threshold $($ RXThresh $=3.65 \mathrm{e}-10 \mathrm{~W})$. The figure 3 and 4 shows the reduction in number of link breaks. EAEDSR reports significantly lesser number of link breaks as compared to original DSR implementation. The results presented are under ideal network conditions and might differ marginally in real situations.

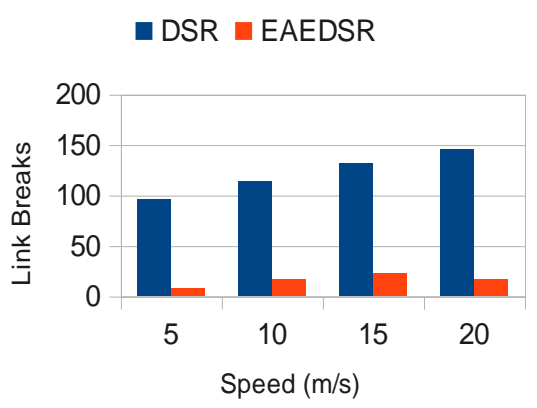

Figure 3: Link Breaks Vs Speed



Figure 4: Link Breaks Vs Number of Nodes

\section{CONCLUSION AND FUTURE WORK}

The proposed technique considers both the link stability and node stability. The network lifetime is reduced either because of node moving out of the radio range leading to link break or node drained of its energy resulting in network partitioning. The proposed metric (LSM) measures the stability of the path and considers both the factors i.e. the node stability and the link duration and is used as the preemption criterion. The links stability reduces with the time and reaches to particular value (threshold) where preemption takes place. Thus the cost of handling the link break is saved. Although the technique may introduce some delay (due to wait period) but the network lifetime is increased. Also the neighbors are selected for broadcasting based on stability and traffic level criterion, hence routing overhead is also reduced. The route selected satisfies the energy aware stability and traffic load constraint, comply with the QoS (quality of service) objectives. The increased delay introduced by wait period may be compensated by using the proposed technique as multipath routing protocol. The simulation results show that proposed EAEDSR performs well and overall can reduce the overhead and link breaks.

\section{REFERENCES}

[1]. D.D. Perkins, H. D. Hughes and C. B. Owen, "Factors Affecting the Performance of Ad Hoc Networks", Proc. of the IEEE International Conference on Communications (ICC) 2002, pp: 2048-2052.

[2]. I. Chlamtac, M. Conti and J.J. N. Liu, "Mobile Ad hoc Networking Imperatives and Challenges", Ad Hoc Networks, Vol 1, 2003, pp: 13-64.

[3]. Sharvani G S, Dr. T.M. Rangaswamy, Nabendu Chaki et al., "Efficient Packet Delivery Approach for Adhoc Wireless Networks," (Eds.): NeTCoM 2010,CSCP 01, 2011, pp. 42-49, 2011.

[4]. B R Sujatha, M V Satyanarayana, "Improved Network Connectivity in MANETs," International Journal of Computer Networks \& Communications (IJCNC), Vol.I, no.3, October 2009.

[5]. Tom Goff, Nael B. Abu-Ghazaleh, Dhananjay S. Phatakand Ridvan Kahvecioglu, "Preemptive Routing in AdHoc Networks," ACM SIGMOBILE 7/01 Rome, Italy, June 2002.

[6]. Sujatha P. Terdal, V. D Mytri, and A Damodaram, "A Preemptive Multipath Routing Protocol for Mobile Ad Hoc Networks," International Journal of Computer Science and Communication Technologies, vol. II, no. 1, July 2009

[7]. Manoj Kumar Singh, Brajesh Kumar, Chiranjeev Kumar and Manish Gupta, "Preemptive MultipathAdhoc on Demand Distance Vector Routing Protocol," International Journal of Computer Science and Information Technology, vol I, no.1, Jan 2011, pp. 3640.

[8]. Sofiane Boukli Hacene, Ahmed Lehireche, Ahmed Meddahi, "Predictive Preemptive Ad Hoc On Demand Distance Vector Routing," Malaysian Journal of Computer Science, Vol. 19 (2), 2006.

[9]. Wenbo Zhu, Xinming Zhang, Yongzhen Liu, Nana Li, "Improve Preemptive Routing Performance in Mobile Ad hoc Networks with Cache-enabled Method," Proc. of International Conference on Communications and Networking in China (Chinacom), August 2008.

[10]. Srinath Perur, Abhilash P. and Sridhar Iyer, "Router Handoff: A Preemptive Route Repair Strategy for AODV," proc. of the IEEE International Conference on Personal Wireless Communications, New Delhi, India, 15-17 December 2002, pp.168-171.

[11]. M. Bheemalingaiah, M. M. Naidu, D. Sreenivasa Rao \& G. Varaprasad (2009), “ Energy Aware Node Disjoint Routing in Mobile Ad Hoc Networks", Journal of Theoretical and Aplied Information Technology, pp: 416-431.

[12]. Zhengyu W, Xiangjun D and Lin C, “ A Grid Based Energy Aware Node-Disjoint Multipath Routing Algorithm ofr MANETs", Proceedings of International Conference on Natural Computation, Vol. 5, pp: 244248. 
[13]. Do-Youn H, Eui-Hyeok K and Jae-Sung L, "An Energy Aware Source Routing with Disjoint Multipath Selection for Energy- Efficient Multihop Wireless Ad hoc Networks", Proceedings of International Federation for Information Processing, 2006, pp: 41-50.

[14]. M. Maleki, K. Dantu and M. Pedram, "Power Aware Source Routing Protocol for Mobile Ad Hoc Networks", Proceedings of the IEEE international symposium on low power electronics and design, pp: 7275.

[15]. Lian Qin, and T Kunz, "Pro-Active Route Maintenance in DSR", ACM SIGMOBILE Mobile Computing and Communication Review (MC2R), Vol. 6, 2001, pp: 79-89.

[16]. D. Kim, Garcia-Luna- Aceves, J.J. Obrackzka, K. Cano \& J. C. Manzoni, "Routing Mechanism for Mobile Ad hoc Networks Based on Energy Drain Rate", Mobile Computing, Vol. 12, 2003, pp: 161-173.

[17]. D.B. Johnson, D.A. Maltz, and J. Broch, "DSR: The Dynamic Source Routing Protcol for Multi-Hop Wireless Ad Hoc Networks", Ad Hoc Networking, Addison-Wesley, 2001, pp: 139-172.

[18]. AchourRhim, Zbigniew Dziong, "Routing Based on Link Expiration Time for MANET Performance Improvement", Proc. of IEEE Malaysian Internation Conference on Communications, 2009

[19]. Sharvani G.S., Dr. T. M. Rangaswamy, "Efficient Packet Delivery Approach for Adhoc Wireless Networks", Journal of Computer Science and Information Technology, Vol. 1, No. 2, pp: 42-49, 2011.
[20]. The NS-2 Simulator, http://www.isi.edu/nsnam/

[21]. Juan Carlos Cano, Pietro Manzoni, “ A Performance Comparison of Energy Consumption for Mobile Ad Hoc Networks Routing Protocols “,

[22]. Liang Qin, and T. Kunz, "Pro-Active Route Maintenance in DSR", ACM SIGMOBILE Mobile Computing and Communications Review (MC2R), vo. 6, issue 3, 2002, pp. 79-89.

[23]. V. Rishiwal, Mano Yadav, S. Verma and S. K. Bajpai, "Power Aware Routing in Ad Hoc Wireless Networks", Journal of Computer Science and Technology (JCS\&T), Vol. 9, No.2., 2010.

[24].Susmit maity et. al., "Pre-emptive Dynamic Source Routing: A Repaired Backup Approach and Stability with Multiple Routes", JCIT, vol. 16, 2008, pp. 91-99.

[25]. A. Kumar, M.Q. Rafiq and Kamal Bansal, “ A Survey of Link Failure Mechanism and Overhead of Routing Protocols in MANET", International Journal of Computer Science and Information Technologies, Vol. 2 (5), pp. 2421-2425, 2011.

[26].Dr. S. Upadhyay, Charu Gandhi, "Node Disjoint Multipath Routing Considering Link and Node Stability Protocol: A Characteristics Evaluation", International Journal of Computer Science, Vol. 7 (1), No. 2, 2010.

[27]. A. Kumar, M. Q. Rafiq, Kamal Bansal, "Performance Evaluation of Energy Consumption in MANET", International Journal of Computer Applications, Vol. 42, No. 2, 2012, pp: 7-12. 